\title{
ANALYSIS OF COMMON QUALITY PROBLEMS IN COORDINATE TRANSFORMATION BETWEEN REFERENCE COORDINATE SYSTEM AND GEOCENTRIC COORDINATE SYSTEM
}

\author{
Zhang Xunhu ${ }^{1}$, Zhang Jixian ${ }^{1}$, Zhang Lei ${ }^{1}$, Liu Jinhu ${ }^{1}$ \\ 1 National Quality Inspection and Testing Center for Surveying and Mapping Products, Beijing 100830, China - 8893784@qq.com; \\ zhangjx@casm.ac.cn; 1848358224@qq.com; 94586053@qq.com
}

KEY WORDS: Reference coordinate system, Geocentric coordinate system, Coordinate transformation, Common quality problems, $4 \mathrm{D}$ product

\begin{abstract}
:
In the working of the existing reference coordinate system transformed into the coordinate system of the geocentric coordinate system, many experts and scholars have in-depth research on the transformation methods such as geodetic control point results (including GNSS base station coordinates, GNSS control point coordinates, triangle point coordinates), basic geographic information data results (including DLG, DOM, DEM, DRG, DSM , etc.) and other spatial information data. On the basis of these studies, many provinces and cities have completed the coordinate system transformation of surveying and mapping results in China. This paper expounds the method of transforming the existing reference coordinate system into the geocentric coordinate system, summarizes and sorts out the common quality problems in the process of different data and different methods, and analyses the causes of the problems and the impact on the transforming results. Based on the above work, The paper provides reference and suggestions for the transforming work, which is aimed to improve the transformation quality in the future.
\end{abstract}

\section{INTRODUCTION}

Before the 1950s, in order to study the shape of the local earth's surface, we reduce the ground measurement data to the minimum correction of the ellipsoid and select the reference ellipsoid and the land of the region where it is located. Under the conditions of the best fit of the level surface, the respective local geodetic coordinate systems are established, such as Pulkovo 1942, North American Datum 1927, Puerto Rico Datum, 1954 Beijing coordinate system, 1980 Xi'an coordinate system, etc. The geocentric geodetic coordinate system with the centre of mass of the Earth as the origin and the ellipsoid positioning closest to the global geoid is the basic geodetic coordinate system that is universal in the 21 st century, such as PZ-90 (Parametry Zemli 1990), WGS-84(World Geodetic System-1984 Coordinate System), CGCS2000(China Geodetic Coordinate System 2000). Since entering the space age of the 21 st century, the Earth's centroid coordinate system with the origin of the Earth's weight centre has completely replaced the local best-fitted reference coordinate system. In order to preserve and utilize existing spatial information data, and ensure the research and use of these data in future work, it is necessary to transformation the existing reference coordinate system results into the geocentric coordinate system results. Spatial information data that needs to be converted includes control point results, digital mapping results, and spatial database results. All case in this paper are transformation between 1954 Beijing coordinate system or the 1980 Xi'an coordinate system and the China Geodetic Coordinate System 2000 .

Since July 1, 2008, China has used the CGCS2000. Since July 1, 2018, the competent departments of surveying and mapping geographic information no longer provide spatial information results of non-China Geodetic Coordinate System 2000s. Relevant departments have carried out the transformation of existing surveying and mapping production to the CGCS2000. The transformation results mainly include the following two aspects:

1. Geodetic control points, such as Global Navigation Satellite System (GNSS) base stations, GNSS control points, triangular points.

2. Basic geographic information data and other spatial information data, such as Digital Line Graphic (DLG), Digital Orthophoto Map (DOM), Digital Elevation Model (DEM), Digital Raster Graphic (DRG), Digital Surface Model (DSM), etc.

Professor Chen, J.Y. and Ning, J.S. totally introduced the construction, refinement, maintenance, and updating of the CGCS2000, and also introduced the national-level surveying and mapping production which can be used for the transformation work. Professor Cheng,Y.Y., Guo,C.X., Lü,Z.P., Wang,W.L. and Pang,S.Y. studied the transformation methods of GNSS reference stations, geodetic control points, and 4D products. Professor Cheng, P.F., explores a method to inspecting the coordinate transforming results. Zhang, X.H. explores a method for quality inspection of coordinate transformation results.

This paper first introduces the transformation of the Geodetic control points results and the transformation of basic geographic information results, and then analyses the common quality problems of the results in the coordinate system transformation process. Based on the analysis of the causes of common quality problems in coordinate system transformation results, the corresponding solutions for various problems are given. 


\section{METHOD OF COORDINATE TRANSFORMATION}

\subsection{Geodetic Control Points}

2.1.1 Method of Coordinate Reduction: In this method, the speed of the station is calculated according to the speed of the plate movement, and the site coordinates are reduced from one epoch to another epoch according to the calculation speed. The coordinate reduction is applicable to the coordinate transformation of the provincial GNSS reference station and the satellite earth control point coordinates from non-CGCS2000 to the CGCS2000. The points to be converted are jointly measured with the surrounding stable IGS station and the national GNSS continuous operation base station. Through above measured, we can obtain the position of each station under the current ITRF framework and observation epoch. Then we can calculate the coordinate. The coordinate calculation consists of three steps, which are epoch reduction, plate motion correction and framework transformation. To convert in this way, you must know the speed field of each station in the network.

2.1.2 Method of Parameter transformation: This method uses the applicable transformation model and transformation parameters to transformation the coordinates of the earth control points from one coordinate system to another. This method is suitable for coordinate transformation of satellite earth control points and triangle point coordinates using the same name control point coordinates in two coordinate systems of known points. That is, according to the transformation area, select the appropriate transformation model (Bursal model, threedimensional seven-parameter model, two-dimensional sevenparameter model, three-dimensional four-parameter model, twodimensional four-parameter model, polynomial fitting model, etc.), select coincidence point, then eliminating the gross errors, calculating the transformation parameters, and coordinate transformation is performed.

\subsection{Basic geographic information data and other spatial information data}

The Basic geographic information data and other spatial information data transformation is performed for the framing data or the database entity data, and different coordinate transformation methods are adopted.

2.2.1 Method of Grid point coordinate transformation correction calculation: Generally, partitioning is performed according to $\left(2^{\circ} \times 3^{\circ}\right)$, and each partition is expanded outward by about $20^{\prime}$, and the transformation parameters of each partition are respectively calculated, and then the $B$ and $L$ of the grid points are respectively calculated by using the determined transformation method and the transformation model. Further, the difference $D B, D L$ between the original coordinate system of each point and the new geodetic coordinate system is obtained, and the transformation correction amount $D B, D L$ of the grid point is formed.

$$
\begin{aligned}
& D B=B_{N}-B_{O} \\
& D L=L_{N}-L_{O}
\end{aligned}
$$

Where $B_{N}$ is the latitude under the converted geodetic coordinate system $B_{O}$ is the latitude in the original coordinate system.
$L_{N}$ is the longitude under the converted geodetic coordinate system

$L_{O}$ is the longitude in the original coordinate system.

For the coordinate system results without the overall adjustment, it is necessary to calculate the geodetic coordinate corrections $d B$ and $d L$ according to the formulas (3) and (4), and then calculate the geodetic coordinates of each point according to the formulas (5) and (6). Then, the Gaussian projection formula is used to calculate the corresponding Gaussian plane coordinate system coordinates, and then calculate the transformation correction $D X_{1}, D Y_{1}$ and the difference correction amount $D X_{2}, D Y_{2}$ of each network point, calculate the correction amount $D X, D Y$ of the new and old coordinate system transformation according to formulas (7) and (8), and finally convert $D X$, $D Y$ into Grid point geodetic coordinate transformation correction $D B_{1}, D L_{l}$.

$$
\begin{aligned}
& \mathrm{dB}=-\frac{\Delta \mathrm{x}}{\mathrm{M}} \sin \mathrm{B}_{\mathrm{O}} \cos \mathrm{L}_{\mathrm{O}}-\frac{\Delta \mathrm{y}}{\mathrm{M}} \sin \mathrm{B}_{\mathrm{O}} \sin \mathrm{L}_{\mathrm{O}}+\frac{\Delta \mathrm{z}}{\mathrm{M}} \cos \mathrm{B}_{\mathrm{O}} \\
& +\frac{1}{\mathrm{M}}\left[\frac{\mathrm{e}^{2}}{\mathrm{~W}} \Delta \mathrm{a}+\frac{\mathrm{N}}{2 \mathrm{~W}^{2}}\left(2-\mathrm{e}^{2} \sin ^{2} \mathrm{~B}_{\mathrm{O}}\right) \Delta \mathrm{e}^{2}\right] \sin \mathrm{B}_{\mathrm{O}} \cos \mathrm{B}_{\mathrm{O}} \ldots \\
& \mathrm{dL}=-\frac{1}{\mathrm{~N} \cos \mathrm{B}_{\mathrm{O}}}\left(\Delta \mathrm{x} \sin \mathrm{L}_{\mathrm{O}}-\Delta \mathrm{y} \cos \mathrm{L}_{\mathrm{O}}\right)
\end{aligned}
$$

Where $L_{O}$ and $B_{O}$ is the longitude and latitude in the original coordinate system.

$$
\begin{aligned}
& \Delta \mathrm{a}=\mathrm{a}_{N}-\mathrm{a}_{O} \\
& \mathrm{a}_{N} \mathrm{a}_{O} \text { are the long radii of the transformed ellipsoid } \\
& \text { and the ellipsoid of the pre-conversion coordinate } \\
& \text { system, respectively. } \\
& \Delta e^{2}=e_{N}^{2}-e_{O}^{2} \\
& e_{N} e_{O} \text { are the first eccentricity of the transformed } \\
& \text { ellipsoid and the ellipsoid of the pre-conversion } \\
& \text { coordinate system, respectively. } \\
& B=B_{O}+d B \\
& L=L_{O}+d L \\
& D X=D X_{1}+D X_{2} \\
& D Y=D Y_{1}+D Y_{2}
\end{aligned}
$$

Calculate the coordinate transformation correction $D B_{2}$ and $D L_{2}$ according to formulas (9) and (10) using $D B$ and $D L$ calculated by formulas (1) and (2).

$$
\begin{aligned}
& D B_{2}=D B+D B_{1} \\
& D L_{2}=D L+D L_{1}
\end{aligned}
$$




\subsubsection{Method of DLG database transformation:}

1. Using the coordinate correction of the four contour points of each frame, the coordinate correction of each element point in the frame is calculated by bilinear interpolation, and the coordinate correction of each element point is used to calculate the coordinate of each element in the new coordinates system.

2. Complete the stitching with the surrounding frames.

3. Complete profile changes, data editing, band-joining, topology reconstruction.

4. Make changes to content such as spatial data metadata.

5. Complete data post-processing such as database warehousing.

\subsubsection{Method of DEM and DSM database} transformation: The data transformation is completed by using the vector data formed in the DEM production process and the DEM discrete point data.

1. Select the coordinate correction of each of the four map points of each frame, calculate the coordinate correction of each element point in the frame by bilinear interpolation, and complete the coordinate conversion of each element point in the frame, and the surrounding frames are stitched together.

\section{Build the TIN}

3. Interpolate DEM or DSM according to relevant specifications or regulations.

\section{Modify the metadata and other content.}

2.2.4 Method of DOM and DRG database transformation: The data transformation is performed using a grid-by-grid correction method while maintaining the original resolution.

1. In the converted geodetic coordinate system, the contour coordinates and the kilometre grid are generated, and the grid points are corrected by the grid.

2. Modify metadata related items and related extra-text decorations.

\section{ANALYSIS OF COMMON QUALITY PROBLEMS}

\subsection{Quality problems in Geodetic Control Points}

3.1.1 Coordinates are reduced: The key processes for coordinate transformation using coordinate reduction methods are: selection of reference control points (IGS stations, national GNSS reference stations, national GNSS geodetic control points), high-precision data processing, plate motion correction, and frame transformation. The common problems of coordinate reduction are mainly concentrated on the selection of reference control points and the correction of plate motion.

1. Calculation of coordinates CGCS2000 coordinates issued by the authority of the non-national surveying and mapping administrative department.

In one group of provinces, the CGCS2000 starting point coordinates used by the basic control network are issued by the authority of the non-national surveying and mapping administrative department, or the energy value is traced back to the authoritative data of the national surveying and mapping administrative department.

Table 1 shows the difference statistics between the CGCS2000 coordinates of the adopted IGS station or the national GNSS reference station and the national authoritative data. This difference leads to systematic deviation of the conversion data of the C-class GNSS control network in X province.

\begin{tabular}{|c|c|c|c|c|}
\hline No. & $\begin{array}{c}\text { X Medium } \\
\text { error X }\end{array}$ & $\begin{array}{c}\text { Y Medium } \\
\text { error Y }\end{array}$ & $\begin{array}{c}\text { Z Medium } \\
\text { error Z }\end{array}$ & $\begin{array}{c}\text { Point } \\
\text { Medium } \\
\text { error }\end{array}$ \\
\hline 1 & -0.003 & -0.049 & -0.128 & 0.137 \\
2 & -0.035 & -0.063 & -0.161 & 0.176 \\
\hline
\end{tabular}

Table 1 Statistics of the starting point coordinates (unit: $\mathrm{m}$ )

2. Calculation points accuracy level does not meet the corresponding level control point requirements.

When some provinces and cities coordinate reference framework or basic control network is established, only the B, $\mathrm{C}$ and D-level GPS control networks with lower accuracy levels in the provinces and cities and surrounding provinces, such as the coordinates of the 2000 national GPS geodetic control network, are used as the starting data. No joint measurement with IGS station or national GNSS reference station, CGCS2000 coordinates obtained by constrained adjustment. This approach ensures consistency of results with other outcomes in the province's region, and the rapid adoption of CCCS2000 was achieved under historical conditions and during excessive periods. Because the provincial GNSS reference station serves as the backbone and main support of the provincial geodetic reference, the coordinate transformation results achieved by this method are not conducive to maintaining the provincial three-dimensional and dynamic geocentric coordinate system, which is not conducive to ensuring the three-dimensional geocentric coordinate's accuracy and current situation and national unity .of the geodetic control network.

Table 2 is the externally coincident precision of a provincial GNSS base station. In the table, 56 provincial GNSS reference stations are based on B and C-level GPS points as the starting point, and are converted by means of coordinate reduction. It can be clearly seen that there are certain dimensions Errors in the three-dimensional direction of space, and all have systematic deviations. These errors have already had an impact on the service of the GNSS base station.

\begin{tabular}{|c|c|c|c|c|}
\hline No. & $\mathrm{X}$ & $\mathrm{Y}$ & $\mathrm{Z}$ & $\mathrm{S}$ \\
\hline Minimum & -0.044 & 0.061 & 0.045 & 0.080 \\
Maximum & -0.019 & 0.080 & 0.059 & 0.105 \\
Average & -0.031 & 0.071 & 0.052 & 0.093 \\
Medium error & \pm 0.031 & \pm 0.071 & \pm 0.052 & \pm 0.094 \\
\hline
\end{tabular}

Table 2 Statistics of the externally coincident precision of a provincial GNSS base station outside the network (unit: $\mathrm{m}$ )

3. Coordinate change values due to plate motion between different epochs of the frame are not considered. In a second group of provinces, after the epoch reduction, the coordinates of the epoch corresponding to the calculation framework are not 
considered. From the observation epoch to the transformation epoch, the coordinate change value caused by the plate motion is brought into the transformation result and causing transformation error

The speed field of the station when ITRF 2005 is converted to the ITRF 2000 framework plays a major role, so if the determined speed field is not accurate, it will have a great impact on the transformation result. The main role of the transition from ITRF 2000 to ITRF 97 is the transformation relationship between the frameworks. The speed field requirements for the stations that need to be converted are not very high.

Table 3 shows the joint adjustment of the observation data of some points in different years. Because the coordinate change value caused by the plate motion cannot be eliminated, resulting in systematic errors in the final transformation results.

\begin{tabular}{|c|c|c|c|c|c|}
\hline No. & Date 1 & Date2 & $\Delta \mathrm{X}$ & $\Delta \mathrm{Y}$ & $\Delta \mathrm{Z}$ \\
\hline 1 & $2005-9$ & $2010-9$ & -0.0119 & +0.0444 & +0.0292 \\
2 & $2005-9$ & $2010-9$ & -0.0018 & +0.0569 & +0.0535 \\
3 & $2003-8$ & $2005-9$ & +0.0151 & +0.0519 & +0.0509 \\
4 & $2003-8$ & $2005-9$ & +0.0007 & +0.0002 & -0.0081 \\
5 & $2003-8$ & $2005-9$ & -0.0037 & +0.0027 & +0.0039 \\
\hline
\end{tabular}

Table 3 Differences in coordinates of the same name in different years (unit: $\mathrm{m}$ )

\subsubsection{Transformation use Parameter}

The key processes of coordinate transformation using parameter conversion method include: transformation ` partition, selection of transformation model, selection and elimination of coincidence points, calculation of transformation parameters, external inspection and so on. The common quality problems of parameter transformation using Parameter mainly focus on four aspects: transformation partition, coincidence point coverage, coincidence point elimination, and checkpoint distribution.

1. Since the coordinates of the Beijing coordinate system in 1954 were calculated by the method of stepwise control of the divisional adjustment, there is obvious adjustment of the adjustment, and even in some areas, the results of the division or the combination of the lock and the net have inconsistent or cracks. Therefore, it is not appropriate to use a partition and a set of transformation parameters in such areas to avoid large errors.

2. The basic principle of coincidence point selection is high level, high precision, small local deformation, uniform distribution, covering the entire transformation area. When the coincidence point cannot cover the entire transformation area, the area transformation parameters that cannot be covered can only be obtained by extrapolation, but the transformation precision may be sharply lost with the extrapolation distance amplification, resulting in the situation that the converted result is not connected with the neighbouring results. For the astronomical geodetic points of the first, second, third and fourth places with CGCS2000 coordinates applied by the state, all of them are used for the calculation of the transformation model, resulting in the inconsistent level and precision of the coincidence point utilization, and the transformation accuracy is not high. Local deformation is large.
3. Due to the gross error of some starting points, and when elimination the gross error point is not strict in the calculation process, it is not strictly performed in the error of more than 3 times, which is easy to cause deformation of local conversion parameters. The elimination of the rough point should also include points that cause uneven distribution of coincidence points. For example, the occurrence of narrow triangles in the model should be avoided to the maximum, and such points can be used as external checkpoints.

4. Using the coincidence point that is not involved in the calculation of the transformation parameters as the external checkpoint, the number of points should be no less than 6 and evenly distributed. When the external checkpoint is insufficient, the field test should be carried out, especially paying attention to the check of the edge of the transformation area.

\subsection{Quality problems in Basic geographic information data results and other spatial information data}

3.2.1 DEM transformation: Since the process data (contour lines, characteristic lines, elevation points, etc.) for the production of DEM results generally do not exist, the DEM transformation cannot construct the TIN interpolation to regenerate the DEM according to the relevant production technical regulations, and generally adopts the high-resolution grid coordinate correction amount. The method of translation or bilinear interpolation performs coordinate transformation on the frame, and at the same time, the reference coordinates are determined by reference pixel resolution for data resampling, and the frame is cut according to the new contour of CGCS2000 and overlapping pixels, and the positioning in the data header file is changed. Coordinates, modify metadata related entries. The main problems of DEM transformation are as follows.

1. Using the translation method to perform DEM transformation, the average value of the four corner points of the image is used as the correction point in the lower left corner of the frame. Without data resampling, the DEM data is still stored in the original coordinate system.

In this method, the coordinates of the starting point of the frame are integer multiples of the non-grid spacing, and the frame is not edged due to the inconsistent translation of the adjacent frame coordinates. In the later application of DEM data, the elevation in the edge area still needs to be processed, and the resampling accuracy is lost.

2. The DEM transformation is performed by the translation method, and the coordinate translation amount is normalized to an integer multiple of the DEM grid spacing, and data resampling is not performed.

This method will produce a row (one column) of DEM grid points between the adjacent frames of the DEM, resulting in a grid number at the edge of the local frame is not unique and there is one row (one column) is missing or one row (one column) is coincident( Figure 1). Because the coordinate translation gauge is normalized to an integer multiple of the grid point spacing, the DEM transformation accuracy is lost, and the transformation accuracy is exceeded. 


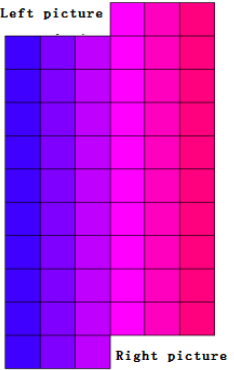

Norma1 edge

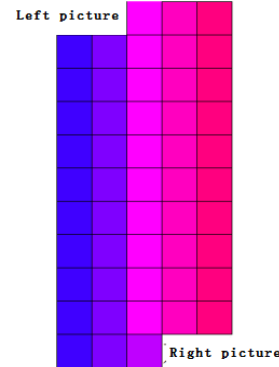

Missing a column

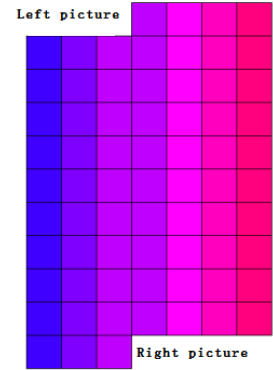

Right picture
Figure 1 A difference between adjacent DEM frames after transformation

Table 4 shows the DEM transformation accuracy statistics of different terrain categories in a certain province. It can be seen that this method is likely to cause partial frame transformation accuracy to exceed the limit in mountainous areas.

\begin{tabular}{|c|c|c|c|}
\hline Terrain category & $\begin{array}{c}\text { Number of } \\
\text { checkpoints }\end{array}$ & Limit $(\mathrm{m})$ & $\begin{array}{c}\text { Medium } \\
\text { error }(\mathrm{m})\end{array}$ \\
\hline Mountain/Alpine & 114 & 2.00 & 0.707 \\
Mountain & 110 & 1.00 & 1.001 \\
Mountain & 114 & 1.00 & 1.257 \\
\hline
\end{tabular}

Table 4 1: 10000 DEM transformation accuracy statistics

3.2.2 DOM transformation: DOM transformation generally uses high-resolution grid coordinate correction. The translation is performed by the translation or correction method. The frame is cut according to the new contour of CGCS2000 and overlapping pixels, and the movement amount (pixel number) is calculated by pixel relationship. , change the positioning coordinates in the data header file, modify the metadata related entries. The main problems of DOM transformation are as follows.

During the DOM transformation process, the translation gauge is normalized to an integer multiple of the DOM ground sampling interval, and then the coordinates of the whole image are translated, resulting in a row (one column) of DOM grid points between the adjacent frames, resulting in local adjacent frames. The edge area values are not unique. Although this transformation method does not have a significant impact on the DOM transformation accuracy, but the transformation work has not been fully completed, it is advisable to resample the results of the joint edge and improve the transformation work.

\section{SUGGESTION}

\subsection{Geodetic Control Points}

The result of the control point CGCS2000 in the process of adjustment calculation can not only use the 2000 national GPS geodetic control network results (three network adjustment results, the average accuracy of geocentric coordinates is better than $\pm 3 \mathrm{~cm}$ ) As a result, it is necessary to make full use of the GNSS continuous operation base station coordinates (coordinate accuracy of millimetres) in the 2000 national GPS geodetic control network with higher precision.
4.1.1 Taking into account the characteristics of plate motion and the strict transformation relationship between different epoch frames in the process of coordinate reduction, making full use of the state-level latest velocity field result CGCS2000 plate motion model (China Plate Model-CGCS2000, CPMCGCS2000) and CGCS2000 grid velocity field model. CPMCGCS2000 is the most accurate correlation model in China. It solves the problem of dynamic maintenance of CGCS2000 and the transformation of basic mapping results in China. It is suitable for the transformation of various GNSS positioning results to CGCS2000 based on the ITRF framework.

4.1.2 Each province and neighbouring provinces exchange data of coincidence points, and the coincidence points of the parameter transformation model are established to cover all transformation areas as much as possible; the transformation area transformation parameters that cannot be covered can be obtained by smooth extrapolation, but external inspection work and the neighbouring province's border work should be strengthened.

4.1.3 Using different transformation models for comparative analysis, plot the point residual distribution map and the point residual contour map, and select the optimal model for coordinate transformation. The polynomial fitting model is used for coordinate transformation. It is also necessary to refer to the Bursa model, the two-dimensional seven-parameter transformation model and other transformation models suitable for the national and provincial scopes for accuracy analysis, and eliminate the larger residuals.

\subsection{Basic geographic information data results other spatial information data}

The existing results transformation workload is large, and the existing basic geographic information data results other spatial information data of the provinces are transitional results, which should be transformed in different ways according to the purpose of the application of the results.

4.2.1 With the continuous development of basic surveying and mapping work, the results of new basic geographic information data should be directly produced by CGCS2000.

4.2.2 If the result of the transformation is indeed required, the data should be transformed according to the corresponding standards of the coordinate system transformation, based on the comparison analysis of multiple transformation schemes, using a strict and complete transformation process. The integrity, consistency and uniqueness of the converted data should be guaranteed during the transformation process to ensure that the transformation is in place and the edges are in place.

4.2.3 Strengthen the comprehensive quality assessment of the technical and technical routes of the transformation work to avoid major quality problems in data transformation.

\section{CONCLUSION}

The transformation of the existing reference coordinate system mapping results to the geocentric coordinate system is a systematic work. In the actual transformation work, although a series of theoretical research and actual transformation work were carried out on the actual situation of the transformation results, some quality problems were still found in the quality inspection of the transformation results. In this paper, the 
quality problems in the transformation results are analysed, the causes of the problems are analysed, and the methods to solve the problems are proposed. These methods are applied to the coordinate transformation work of 9 provinces and 3 cities, avoiding the corresponding quality problems. Among them, the transformation results of three provinces modified the transformation plan according to the solution proposed in the paper, and the results were re-transformed. After re-examination of the transformation results, the results have reached a good quality level, and the method proposed in the paper is reasonable and feasible. Therefore, the solution to the problem proposed in the paper is suitable for coordinate system transformation work.

\section{REFERENCES}

Chen, J.Y., Yang, Y.X., 2007a. Construction of 2000 National Geodetic Control Network and Its Technological Progress. Journal of Surveying and Mapping, 01:1-8.

Cheng, P.F., Cheng, Y.Y., Wen, H.J., 2008. 2000 National Geodetic Coordinates Practical Collection. Beijing, Surveying and Mapping Press, 130-132.

Cheng, Y.Y., Cheng, P.F., Mi, J.Z., 2011a. Transformation of provincial and municipal CORS stations to CGCS2000 based on the current framework. Bulletin of Surveying and Mapping, 2011, 07:1-3+14.

Cheng, Y.Y., Cheng, P.F., Gu,D.S.,2010a. Transformation of map to CGCS2000 by three-dimensional 4-parameter model. Journal of Wuhan University (Information Science Edition), 06: $747-751+755$.

Cheng, Y.Y., Cheng, P.F., Mi, J.Z., 2007a. Study on the transformation method of $1980 \mathrm{Xi}$ 'an coordinate system and WGS84 coordinate system in large-scale spatial domain. Bulletin of Surveying and Mapping, 12: 5-8.

Dong, H.W., 2001a. Historical Notes of the Beijing Coordinate System in 1954. Northeast Surveying and Mapping, 02: 16$17+23$.

Guo ,C.X., Wang, W.L., Bai,G.X.,2013a. Determination of National High-precision High-resolution Grid Correction in Coordinate System Transformation. Sci-Tech Scientific, 02: 5-7.

Guo, C.X., Han, M.X., 2013. Coordinate transformation method of digital elevation model (DEM) and digital line graph (DLG). Bulletin of Surveying and Mapping, 01:57-59.

Han, M.X., Guo, C.X., Wang, W.L., 2014a. The method of transforming the basic data of small scale from 1980 coordinate system to 2000 coordinate system. Surveying and Mapping Science, 01: 32-34+58.

Lü, Z.P., Wei, Z.Q, Li, J., 2013a. Establishment of CGCS2000 high precision coordinate transformation grid model. Journal of Surveying and Mapping, 06: 791-797.

Ning, J.S., Wang, H., Cheng, P.F., 2015a. Construction and Progress of China Geodetic Coordinate System 2000 Framework System. Journal of Wuhan University (Information Science Edition), 05: 569-573.
NIMA TR8350.2, 2000b. Department of Defense World Geodetic System 1984. Amendment1.3 January 2000.

Pang, S.Y., Guo, C.X., Cheng, C.L., 2006a. Research on Coordinate Transformation Method of National Basic Scale DLG Data .Science and Mapping Science, S1:28-29.

Shi, J.P. 2010b. Granskning av Earth Coordinate System i USA . Kinesiska samhället för kartläggning och kartläggning, 2010: 7.

Wang, W.L., Cheng, C.L., Li, D., 2012a. Accuracy analysis and applicability of the 1954 Beijing coordinate system and 1980 $\mathrm{Xi}$ 'an coordinate system. Journal of Geodesy and Geodynamics, $05: 68-71+77$.

Zhang, X.H., 2015a. Discussion on the transformation of existing achievements into China Geodetic Coordinate System 2000 results inspection method. Engineering Investigation, 02: 85-89. 